\title{
Lower 30-day readmission rates with roflumilast treatment among patients hospitalized for chronic obstructive pulmonary disease
}

This article was published in the following Dove Press journal:

International Journal of COPD

12 May 2015

Number of times this article has been viewed

\author{
Alex Z Fu' \\ Shawn $X$ Sun $^{2}$ \\ Xingyue Huang ${ }^{2}$ \\ Alpesh $\mathrm{NAmin}^{3}$ \\ 'Georgetown University Medical \\ Center, Washington, DC, ${ }^{2}$ Health \\ Economics and Outcomes Research, \\ Forest Laboratories, LLC, an affiliate \\ of Actavis, Inc., Jersey City, NJ, \\ ${ }^{3}$ Department of Medicine, University \\ of California, Irvine, CA, USA
}

Background: Few data exist related to the impact of roflumilast on health care utilization. This retrospective study estimated 30-day hospital readmission rates between patients who did and did not use roflumilast among those with COPD hospitalizations.

Methods: Data were from MarketScan, a large US commercial health insurance claims database. Patients aged $\geq 40$ years with at least one hospitalization for COPD between 2010 and 2011 were included. The roflumilast group included patients who used roflumilast within 14 days after the first hospitalization (index), while the comparison group (non-roflumilast) included patients who did not use roflumilast during the study period. Continuous enrollment for at least 6 months before and 30 days after the index date was required. The 30-day hospitalization rate was calculated after the index hospitalization. Conditional logistic regression with propensity score 1:3 matching was employed to assess the difference in 30-day hospital readmission rates between the roflumilast and non-roflumilast groups, adjusting for baseline characteristics, comorbidity, health care utilization, and COPD medication use within 14 days after the index date.

Results: A total of 15,755 COPD patients met the selection criteria, ie, $366(2.3 \%)$ in the roflumilast group and $15,389(97.7 \%)$ in the non-roflumilast group. The mean $( \pm$ standard deviation) age was $71 \pm 12.5$ years and $52 \%$ were female. After propensity score matching, all-cause 30 -day hospitalization rates were $6.9 \%$ and $11.1 \%$ in the roflumilast and nonroflumilast groups, respectively. COPD-related 30-day hospitalization rates were $6.3 \%$ and $9.2 \%$ in the roflumilast and non-roflumilast groups, respectively. Conditional logistic regression identified a significantly lower likelihood of all-cause 30-day readmission (odds ratio $0.59,95 \%$ confidence interval $0.37-0.93, P=0.023$ ) for roflumilast patients relative to non-roflumilast patients.

Conclusion: This study showed, in a real-world setting, that use of roflumilast was associated with a lower rate of hospital readmission within 30 days among patients hospitalized for COPD.

Keywords: chronic obstructive pulmonary disease, roflumilast, health care utilization, hospital readmission

\section{Introduction}

COPD is a progressive lung condition characterized by persistent airflow limitation, chronic and progressive dyspnea, cough, and sputum production, and is often complicated by exacerbations. ${ }^{1}$ COPD affects approximately 24 million US adults, including 12 million diagnosed patients and 12 million undiagnosed, ${ }^{2}$ and is the third leading cause of death in the USA. ${ }^{3}$ The annual cost of COPD was estimated at $\$ 49.9$ billion in 2010, with $\$ 29.5$ billion attributable to direct health care costs, in which hospital care cost accounted for the largest share of the total. ${ }^{2}$
Correspondence: Alex Z Fu

Georgetown University Medical Center, 3300 Whitehaven Street, NW, Suite 4I00, Washington, DC 20007, USA

Email zf54@georgetown.edu 
Reducing COPD exacerbations is an important goal of COPD management, given that exacerbations have serious health consequences and are associated with declines in lung function, reduction in health-related quality of life, and increased hospitalization and mortality. ${ }^{4}$ Exacerbation is also one of the primary reasons for the significant economic burden of COPD, as it accounts for up to $45 \%$ of the total cost for treating COPD. ${ }^{4,5}$ Roflumilast, an oral, once-daily, selective phosphodiesterase- 4 inhibitor that reduces moderate and severe exacerbation rates and improves lung function in patients with COPD, ${ }^{6,7}$ is indicated as a treatment to reduce the risk of exacerbations in patients with severe COPD associated with chronic bronchitis and a history of exacerbations. It was approved by the US Food and Drug Administration (FDA) in February 2011.8,9

To better understand the medical care utilization associated with use of roflumilast in the real-world setting, we conducted a retrospective cohort study to compare allcause and COPD-related hospital readmission rates within 30 days between patients who used roflumilast and those who did not.

\section{Materials and methods Data source}

This study used longitudinal, integrated medical and pharmacy claims data from MarketScan databases: Commercial Claims and Encounters (Commercial) and the Medicare Supplemental and Coordination of Benefits (Medicare Supplemental). The MarketScan database includes patient-level, paid and adjudicated medical and pharmacy claims histories of 110 million covered lives belonging to 12 national and regional health plans in the USA. ${ }^{10,11}$ The database captures the full continuum of care in all settings, including physician office visits, hospital stays, and outpatient pharmacy claims. These data are a good representation of the US national, commercially insured population and those who have both Medicare coverage and supplemental employer-sponsored coverage.

In the MarketScan database, each medical service claim has its date of service, International Classification of Diseases, Ninth Revision, Clinical Modification (ICD9-CM) diagnosis codes and procedure codes. There is also the National Drug Code and days of medication therapy supplied information available for pharmacy records. The date-of-service variable is important to identify temporal relationship and define medication treatments.

\section{Study design}

This was a retrospective matched-cohort study using the MarketScan claims database. We included patients aged
40 years or older with at least one hospitalization for COPD, identified as inpatient claims with a COPD diagnosis (ICD9-CM 491, 492, or 496). ${ }^{7}$ The roflumilast patient group was defined as those who had the drug within the first 14 days after the hospitalization for COPD, with the discharge date defined as the index date between July 1 and December 31, 2011. We selected a historic comparison group (non-roflumilast patients), defined as hospitalization for COPD with discharge date (index date) between July 1 and December 31, 2010. Because roflumilast was approved by the FDA in February $2011,{ }^{8,9}$ this approach ensures that the comparison group received no roflumilast treatment following hospitalizations during the study period. The reason we applied a historic comparison group was to reduce confounding by indication, as roflumilast is indicated for exacerbations in patients with severe COPD. ${ }^{7}$

Continuous enrollment of at least 6 months before and 30 days after the index date was required. We excluded patients with cystic fibrosis (ICD-9-CM 277.0x) or lung cancer (ICD-9-CM 162.xx) within the 6 months before the index date (baseline). To minimize misclassification bias, we also excluded patients with hospital readmission prior to their use of roflumilast.

\section{Variables}

We calculated all-cause and COPD-related hospitalization readmission rates within 30 days after the index date. In order to reduce sample selection bias, we adjusted for a list of covariates using a propensity score matching approach. The adjusted covariates included patient demographics, clinical characteristics, and health care utilization at baseline and COPD medication use within a 14-day follow-up period after the index date. Demographic variables included age, sex, geographic region, rural/urban setting, and type of health insurance (Medicare or not). The geographic regions were northeast, north central, west, and south. To control for comorbidities, a Charlson Comorbidity Index ${ }^{12}$ was constructed for each subject based on the ICD-9-CM diagnosis codes of all claims within the 6 months before the index date. We excluded patients with moderate to severe liver disease because roflumilast is specifically contraindicated in these patients and this condition is a category included in the original Charlson Comorbidity Index. We also identified comorbid asthma (ICD-9-CM 493.xx) to further account for this clinically related condition.

To adjust for disease severity, we calculated variables for COPD exacerbations including the total number of severe exacerbation events and the proportion of patients who 
experienced moderate exacerbations within the 6-month baseline period. Exacerbations were identified using medical and pharmacy claims following Lindenauer et al and Mapel et al. ${ }^{13,14}$ A severe exacerbation was defined as a hospital admission for a primary COPD diagnosis with acute exacerbation (ICD-9-CM codes 491.21, 491.22, 493.22, 492.8), or a primary diagnosis of respiratory failure $(518.81,518.82$, 518.84) combined with a secondary diagnosis of COPD with acute exacerbation or emphysema (491.0, 491.1, 491.21, 491.22, 491.8, 491.9, 492.0, 492.8, 493.22, or 496). ${ }^{13} \mathrm{~A}$ moderate exacerbation was identified by: an emergency room visit with the same diagnosis codes as severe exacerbation; at least one medical claim for diagnosis codes of 136.3, 466-466.19, 480-486, 487.0, 490, 491.21, 491.22, 493.02, 493.12, 493.22, 493.92, 494.1, 506.0-506.3, 507-507.8, 511.0-511.1, 512$512.8,517.1,518.0,518.81,518.82,518.84$, and 770.84 in a physician office or outpatient non-emergency room visit; ${ }^{14}$ at least one pharmacy claim for systemic steroids; or at least one pharmacy claim for oral antibiotics commonly used for respiratory infections.

Health care utilization was summarized for each subject, including numbers of all medical service (non-COPD specific) and COPD-related utilizations. Each category included numbers for inpatient, emergency room, outpatient visits, and COPD medications within the 6-month baseline period. COPD medication variables were defined as numbers of medications including long-acting $\beta_{2}$-agonists, long-acting muscarinic antagonists, inhaled corticosteroids, inhaled corticosteroid-long-acting $\beta_{2}$-agonist combinations, or theophylline. Numbers of COPD medication by type were also summarized for the 14 days after the index date, and were adjusted for the propensity score analysis.

\section{Statistical analysis}

Patient demographics, clinical characteristics, health care utilization, and the 30-day post-index hospital readmission rate were compared between the roflumilast cohort and the comparison cohort (non-roflumilast patients). Continuous variables are reported as the mean \pm standard deviation; categorical variables are summarized as percentages. Comparisons were made using appropriate statistical tests (Student's $t$-test for continuous data and Chi-square or Fisher's Exact test for categorical data).

To better balance the characteristics between the roflumilast-treated group and the non-roflumilast comparison group, we used the propensity score matching approach. Variables in the propensity score predictive equation included all patient demographics, clinical characteristics, and health care utilization. We used the greedy matching algorithm ${ }^{15}$ to create the 1:3 matched roflumilast-treated and comparison groups. The matched subsamples were used as the final analytic dataset. We applied conditional logistic regressions to evaluate the odds of having post-index allcause and COPD-related hospital readmissions between the roflumilast and non-roflumilast groups. Conditional logistic regression is used to take into consideration the quadruplets of patients after matching (every roflumilasttreated patient matched with three non-roflumilast comparison patients). ${ }^{16}$

\section{Results}

Table 1 gives the descriptive comparison of characteristics between patients with and without roflumilast before and after the propensity score matching. Based on the inclusion criteria, a total of 15,755 COPD patients, including $366(2.3 \%)$ roflumilast and 15,389 (97.7\%) non-roflumilast patients, were selected. Roflumilast patients were younger (mean $68 \pm 10.9$ versus $72 \pm 11.4$ years), with a higher percentage from the Southern US region (37\% versus 29\%), and a lower percentage under Medicare insurance (53\% versus $71 \%$ ) relative to non-roflumilast patients. Roflumilast patients were more likely to have asthma ( $30 \%$ versus $17 \%$ ) and more severe COPD, represented by severe exacerbation frequency ( 0.45 versus 0.12$)$ and moderate exacerbation $(85 \%$ versus $58 \%$ ) compared with non-roflumilast patients. Health care utilization variables showed that roflumilast patients used more medical services than non-roflumilast patients within the 6-month baseline period (all-cause hospitalizations [0.71 versus 0.44], all-cause ER visits [0.91 versus 0.26], all-cause outpatient visits [20 versus 18], COPD-related hospitalizations [0.58 versus 0.21], COPD-related emergency room visits [0.86 versus 0.24 ], and COPD-related outpatient visits [9.0 versus 4.6]). Roflumilast patients also used more COPD medications both within the 6-month baseline period and the 14-day post-index period. Numbers of these medications were included in the propensity score matching and were presented in Table 1. Percentages of use for these medications were not included in matching and were presented in Table S1.

Propensity score matching resulted in balanced groups in terms of these measured characteristics (Table 1). All of the variables that had statistically significant differences before matching had no significant differences after matching. Roflumilast patients on average had roflumilast $5.8 \pm 4.8$ days after their index hospital discharge. The number of days from the index discharge to hospital readmission was not significantly different between roflumilast and non-roflumilast patients (16.5 \pm 8.1 and $13.3 \pm 8.3$, respectively, $P=0.10$ ) among those 
Table I Patient characteristics between roflumilast-treated group and comparison group

\begin{tabular}{|c|c|c|c|c|c|c|}
\hline & \multicolumn{3}{|c|}{ Before matching } & \multicolumn{3}{|c|}{ After matching } \\
\hline & Roflumilast & Non-roflumilast & $P$-value & Roflumilast & Non-roflumilast & $P$-value \\
\hline $\mathrm{n}$ & 366 & 15,389 & & 350 & 1,050 & \\
\hline \multicolumn{7}{|l|}{ Baseline characteristics } \\
\hline Age at index date, years, mean $\pm S D$ & $67.9 \pm 10.9$ & $71.5 \pm 11.4$ & $<0.0001$ & $68.2 \pm 11.1$ & $68.2 \pm 11.5$ & 0.95 \\
\hline Female & $54.4 \%$ & $52.9 \%$ & 0.58 & $54.0 \%$ & $53.4 \%$ & 0.85 \\
\hline Rural (versus urban) & $20.5 \%$ & $18.9 \%$ & 0.33 & $20.9 \%$ & $21.1 \%$ & 0.91 \\
\hline \multicolumn{7}{|l|}{ Geographic region } \\
\hline Northeast & $17.5 \%$ & $18.1 \%$ & $<0.000$ I & $16.0 \%$ & $17.0 \%$ & 0.06 \\
\hline North central & $32.8 \%$ & $38.3 \%$ & & $34.0 \%$ & $32.5 \%$ & \\
\hline South & $37.2 \%$ & $29.1 \%$ & & $37.4 \%$ & $39.8 \%$ & \\
\hline West & $10.4 \%$ & $14.3 \%$ & & $10.6 \%$ & $10.4 \%$ & \\
\hline Medicare insurance & $52.7 \%$ & $70.8 \%$ & $<0.0001$ & $54.6 \%$ & $55.3 \%$ & 0.80 \\
\hline $\mathrm{CCl}$ & 1.899 & 2.257 & $<0.0001$ & 1.897 & 1.846 & 0.55 \\
\hline Asthma & $29.5 \%$ & $17.0 \%$ & $<0.000$ I & $28.0 \%$ & $29.3 \%$ & 0.63 \\
\hline Severe exacerbation frequency per patient & 0.45 & 0.12 & $<0.0001$ & 0.38 & 0.41 & 0.59 \\
\hline Moderate exacerbation (yes/no) & $85.2 \%$ & $57.5 \%$ & $<0.0001$ & $84.6 \%$ & $88.0 \%$ & 0.10 \\
\hline \multicolumn{7}{|c|}{ COPD medications within 6-month baseline period } \\
\hline LABAs $(n)$ & 0.38 & 0.06 & $<0.0001$ & 0.35 & 0.25 & 0.18 \\
\hline LAMAs (n) & 1.64 & 0.49 & $<0.0001$ & 1.53 & 1.47 & 0.62 \\
\hline ICS (n) & 0.49 & 0.19 & $<0.0001$ & 0.42 & 0.40 & 0.81 \\
\hline ICS-LABA combinations $(n)$ & 1.49 & 0.58 & $<0.0001$ & 1.40 & 1.41 & 0.89 \\
\hline Theophylline medications & 0.33 & 0.09 & $<0.0001$ & 0.30 & 0.26 & 0.55 \\
\hline \multicolumn{7}{|c|}{ Health care utilization within 6-month baseline period } \\
\hline COPD-related inpatient visits $(\mathrm{n})$ & 0.58 & 0.21 & $<0.000$ I & 0.50 & 0.52 & 0.76 \\
\hline COPD-related ER visits (n) & 0.86 & 0.24 & $<0.0001$ & 0.65 & 0.72 & 0.54 \\
\hline COPD-related outpatient visits (n) & 9.03 & 4.64 & $<0.0001$ & 8.47 & 8.50 & 0.96 \\
\hline All medical service inpatient visits ( $n$ ) & 0.71 & 0.44 & $<0.0001$ & 0.63 & 0.65 & 0.79 \\
\hline All medical service ER visits ( $n)$ & 0.91 & 0.26 & $<0.0001$ & 0.70 & 0.77 & 0.53 \\
\hline All medical service outpatient visits (n) & 19.81 & 17.57 & 0.007 & 19.30 & 19.61 & 0.74 \\
\hline \multicolumn{7}{|c|}{ COPD medications within 14 days after the index date } \\
\hline $\operatorname{LABAs}(n)$ & 0.05 & 0.01 & $<0.0001$ & 0.05 & 0.03 & 0.36 \\
\hline LAMAs (n) & 0.26 & 0.07 & $<0.0001$ & 0.23 & 0.22 & 0.66 \\
\hline $\operatorname{ICS}(n)$ & 0.09 & 0.03 & 0.0001 & 0.09 & 0.07 & 0.52 \\
\hline ICS-LABA combinations $(\mathrm{n})$ & 0.24 & 0.09 & $<0.0001$ & 0.23 & 0.24 & 0.73 \\
\hline Theophylline medications ( $\mathrm{n}$ ) & 0.07 & 0.01 & $<0.0001$ & 0.07 & 0.05 & 0.44 \\
\hline
\end{tabular}

Note: The data are presented as the mean or percentage, before and after I:3 propensity score matching.

Abbreviations: $\mathrm{CCl}$, Charlson Comorbidity Index; COPD, chronic obstructive pulmonary disease; ER, emergency room; SD, standard deviation; LABAs, long-acting $\beta_{2}$-agonists; LAMAs, long-acting muscarinic antagonists; ICS, inhaled corticosteroids.

who were readmitted. The all-cause hospital readmission rate within 30 days was significantly lower for roflumilast patients than for non-roflumilast patients $(6.9 \%$ versus $11.1 \%$, $P=0.021)$ within the propensity score matched sample. The COPD-related hospital readmission rate was lower, but did not reach statistical significance $(6.3 \%$ versus $9.2 \%, P=0.086)$.
Conditional logistic regression results for the propensity score matched sample are reported in Table 2. Roflumilast patients had a lower likelihood of all-cause hospital readmission within 30 days than non-roflumilast patients (odds ratio $0.59,95 \%$ confidence interval $0.37-0.93, P=0.023$ ). The regression result for 30-day COPD-related hospital

Table 2 Conditional logistic regressions for all-cause or COPD-related hospitalization within 30 days among the propensity score matched sample $(n=I, 400)$

\begin{tabular}{|c|c|c|c|}
\hline & Odds ratio & $95 \% \mathrm{Cl}$ & P-value \\
\hline All-cause hospitalization within 30 days & 0.59 & $0.37-0.93$ & 0.023 \\
\hline COPD-related hospitalization within 30 days & 0.66 & $0.4 I-1.07$ & 0.089 \\
\hline
\end{tabular}

Notes: Each row represents a separate regression. Independent variable of interest is roflumilast-treated (versus non-treated).

Abbreviations: COPD, chronic obstructive pulmonary disease; $\mathrm{Cl}$, confidence interval. 
readmission shows the same trend, but is not statistically significant (odds ratio $0.66,95 \%$ confidence interval 0.41-1.07, $P=0.089)$.

\section{Discussion}

This study estimated, in a real-world setting, that use of roflumilast is associated with a lower likelihood of all-cause 30-day hospital readmission for patients who were previously hospitalized for COPD. Our study is one of the first to focus on health care utilization outcomes for patients with roflumilast in a commercially insured US population.

Exacerbations of COPD are major clinical events that are associated with hospitalization and risk of dying. ${ }^{4}$ Many exacerbations require hospitalization, and account for a large share of health care expense for COPD patients. ${ }^{2}$ Roflumilast is an anti-inflammatory medication that improves lung function in patients with COPD. In clinical trials, roflumilast reduces the frequency of exacerbations in patients with severe airway obstruction, clinical features of chronic bronchitis, and a history of exacerbations. ${ }^{6,7,17}$ It is also recommended as a second or alternative choice combined with a long-acting bronchodilator in COPD patients at high risk for hospitalization. ${ }^{18}$ Thus, it is sensible to infer that roflumilast may reduce exacerbation-related hospitalizations. However, the evidence from clinical trials may not represent the community population. Results from a pooled data analysis of trials indicated that roflumilast leads to reduction in severe exacerbations, and possibly related hospitalizations. ${ }^{19}$ Another review paper concluded that roflumilast did not reduce the frequency of hospitalization for exacerbations, even in the most favorable trials. ${ }^{20}$ On the other hand, trial results may lack generalizability because they typically enroll a relatively healthier patient population (patients with comorbid conditions are commonly excluded from trials) compared with general practice. Our study fills this research gap by providing real-world empirical results as supportive evidence in a large, national, commercially insured US population and those who have Medicare coverage or supplemental employer-sponsored coverage.

We identified that use of roflumilast is associated with lower likelihood of all-cause 30-day hospital readmission. However, the results for COPD-related readmission did not reach statistical significance, although there was a trend for a decreased rate with roflumilast use. This is primarily due to the shorter time and limited data available after FDA approval for roflumilast when we conducted the analysis. This resulted in a small sample for the roflumilast group. Analysis based on more recent data should provide a larger sample size.
Our analysis has several strengths. First, our data are from a geographically diverse population covered by large employer-sponsored private health insurance programs in the USA, ${ }^{10}$ which makes our study results more generalizable to community practice than trials. Second, the MarketScan data are contributed by employers. As long as these patients remain with the same employer, the data capture the full continuum of care and claims in all settings, even though the patients might switch their health plans. An individual usually stays longer with an employer than with a health insurance plan. Third, we applied a historic comparison group to reduce confounding by indication, as roflumilast is indicated for exacerbations in patients with severe COPD. ${ }^{7}$ Additionally, we selected the comparison group from the same calendar months (July to December) as the roflumilast group in order to minimize the seasonal effect linked to respiratory conditions such as COPD. ${ }^{21,22}$

Some limitations of the study should also be noted. First, we defined roflumilast use within 14 days after inpatient discharge to allow a period of time between use of roflumilast and hospital readmission within 30 days. Our results are also limited to those who survived at least 30 days after a COPD hospital discharge. Patients who started roflumilast after 14 days, died within 30 days, or had follow-up data for less than 30 days were excluded from our study. By design, roflumilast patients could not have been readmitted prior to their first roflumilast prescription (within first 14 days post-discharge), while non-roflumilast patients did not need to meet any prescription requirement within the first 14 days post-discharge. This may lead to immortal time bias. ${ }^{23}$ Nonetheless, this bias is not a concern here because the number of days from the index discharge to hospital readmission was not significantly different between roflumilast and nonroflumilast patients. Second, COPD exacerbations are events in the natural course of the disease characterized by a change in the patient's baseline dyspnea, cough, and/or sputum production that is beyond normal day-to-day variations. ${ }^{24}$ Due to the absence of a clinical measure of COPD exacerbation severity and lung function in the claims dataset, we defined exacerbation following Lindenauer et al and Mapel et al. ${ }^{13,14}$ Third, information on patient race/ethnicity, education, income, and clinical measures such as laboratory values were not recorded in the claims database. Not controlling for these unmeasured covariates may lead to biased estimates for the effect of roflumilast on readmission rate. Fourth, all identified medical conditions were based on ICD-9 diagnosis codes in medical claims. Medical conditions not recorded in the claims (such as under-diagnosis) cannot be identified. 
Meanwhile, consumption of medication was assessed using the pharmacy claims record. Whether a patient actually took the medication cannot be confirmed. Finally, there is no way to identify whether our patients participated in clinical trials involving roflumilast, which could lead to misclassification bias, although such a chance should be low.

In summary, our study showed, in a real-world setting, that use of roflumilast is associated with a lower 30-day hospital readmission rate in patients hospitalized for COPD. Such results complement the clinical trials by providing health care utilization outcomes at the population level. Pharmacotherapies such as roflumilast may be an effective option to decrease hospitalizations for patients with COPD exacerbations.

\section{Disclosure}

AZF and ANA report research support from Forest Research Institute, an affiliate of Actavis, Inc., but received no payment for this publication or writing this manuscript. SXS is an employee of Forest Research Institute, an affiliate of Actavis, Inc. XH was an employee of Forest Research Institute during the conduct of the study.

\section{References}

1. Global Initiative for Chronic Obstructive Lung Disease. Global strategy for the diagnosis, management, and prevention of chronic obstructive pulmonary disease. Available from: http:/www.goldcopd.org/guidelinesglobal-strategy-for-diagnosis-management.html. Accessed January 15, 2014.

2. National Heart, Lung, and Blood Institute. Morbidity and Mortality: 2012 Chart Book on Cardiovascular, Lung, and Blood Diseases. Available at: https://www.nhlbi.nih.gov/files/docs/research/2012_ChartBook_508. pdf. Accessed April 23, 2015.

3. Miniño AM, Xu J, Kochanek KD. Deaths: Preliminary data for 2008. Natl Vital Stat Rep. 2010;59(2):1-52.

4. Andersson F, Borg S, Jansson SA, et al. The costs of exacerbations in chronic obstructive pulmonary disease (COPD). Respir Med. 2002;96(9): 700-708.

5. Vestbo J, Anderson JA, Calverley PM, et al. Adherence to inhaled therapy, mortality and hospital admission in COPD. Thorax. 2009;64(11): 939-943.

6. Calverley PM, Rabe KF, Goehring UM, Kristiansen S, Fabbri LM, Martinez FJ. Roflumilast in symptomatic chronic obstructive pulmonary disease: two randomised clinical trials. Lancet. 2009;374(9691): 685-694.

7. Fabbri LM, Calverley PM, Izquierdo-Alonso JL, et al. Roflumilast in moderate-to-severe chronic obstructive pulmonary disease treated with longacting bronchodilators: two randomised clinical trials. Lancet. 2009; 374(9691):695-703.
8. US Food and Drug Administration. FDA approves new drug to treat chronic obstructive pulmonary disease. Available from: http://www. fda.gov/NewsEvents/newsroom/PressAnnouncements/ucm244989. htm. Accessed April 16, 2015.

9. Baye J. Roflumilast (Daliresp): a novel phosphodiesterase-4 inhibitor for the treatment of severe chronic obstructive pulmonary disease. P T. 2012; 37(3):149-161.

10. Zhao Y, Ash AS, Ellis RP, et al. Predicting pharmacy costs and other medical costs using diagnoses and drug claims. Med Care. 2005;43(1): 34-43.

11. Fu AZ, Chen L, Sullivan SD, Christiansen NP. Absenteeism and shortterm disability associated with breast cancer. Breast Cancer Res Treat. 2011;130(1):235-242.

12. Charlson ME, Pompei P, Ales KL, MacKenzie CR. A new method of classifying prognostic comorbidity in longitudinal studies: development and validation. J Chronic Dis. 1987;40(5):373-383.

13. Lindenauer PK, Pekow PS, Lahti MC, Lee Y, Benjamin EM, Rothberg MB. Association of corticosteroid dose and route of administration with risk of treatment failure in acute exacerbation of chronic obstructive pulmonary disease. JAMA. 2010;303(23):2359-2367.

14. Mapel DW, Dutro MP, Marton JP, Woodruff K, Make B. Identifying and characterizing COPD patients in US managed care. A retrospective, cross-sectional analysis of administrative claims data. BMC Health Serv Res. 2011;11:43.

15. Parsons LS. Reducing bias in a propensity score matched-pair sample using greedy matching techniques. Paper 214-26 presented at the SAS Users Group 26th International Conference, Long Beach, CA, USA, April 22-25, 2001.

16. Mack TM, Pike MC, Henderson BE, et al. Estrogens and endometrial cancer in a retirement community. N Engl J Med. 1976;294(23): 1262-1267.

17. Field SK. Roflumilast, a novel phosphodiesterase 4 inhibitor, for COPD patients with a history of exacerbations. Clin Med Insights Circ Respir Pulm Med. 2011;5:57-70.

18. Van de Griend JP, Marcum ZA, Linnebur SA. A year in review: new drugs for older adults in 2011. Am J Geriatr Pharmacother. 2012;10(4): 258-263.

19. Wedzicha JA, Rabe KF, Martinez FJ, et al. Efficacy of roflumilast in the COPD frequent exacerbator phenotype. Chest. 2013;143(5):1302-1311.

20. [No authors listed]. Roflumilast: doubtful efficacy but clear harms in COPD. Prescrire Int. 2013;22(134):5-9.

21. Jenkins CR, Celli B, Anderson JA, et al. Seasonality and determinants of moderate and severe COPD exacerbations in the TORCH study. Eur Respir J. 2012;39(1):38-45.

22. Vilkman S, Keistinen T, Tuuponen T, Kivela SL. Seasonal variation in hospital admissions for chronic obstructive pulmonary disease in Finland. Arctic Med Res. 1996;55(4):182-186.

23. Suissa S. Immortal time bias in pharmaco-epidemiology. Am J Epidemiol. 2008;167(4):492-499.

24. Global Initiative for Chronic Obstructive Lung Disease. Global strategy for the diagnosis, management and prevention of chronic obstructive pulmonary disease. NHLBI/WHO workshop report. Bethesda: National Heart, Lung and Blood Institute, 2008. Available from: http://www. goldcopd.org/uploads/users/files/GOLDWkshp05Clean.pdf. Accessed April 16, 2015. 


\section{Supplementary material}

Table SI Additional patient characteristics that are not included in the propensity score matching

\begin{tabular}{|c|c|c|c|c|c|c|}
\hline & \multicolumn{3}{|c|}{ Before matching } & \multicolumn{3}{|c|}{ After matching } \\
\hline & Roflumilast & Non-roflumilast & $P$-value & Roflumilast & Non-roflumilast & $P$-value \\
\hline $\mathrm{n}$ & 366 & 15,389 & & 350 & $\mathrm{I}, 050$ & \\
\hline \multicolumn{7}{|c|}{ COPD medications within 6-month baseline period } \\
\hline LABA use & $11.5 \%$ & $2.5 \%$ & $<0.0001$ & $11.1 \%$ & $8.0 \%$ & 0.07 \\
\hline LAMA use & $58.2 \%$ & $20.3 \%$ & $<0.0001$ & $56.9 \%$ & $45.7 \%$ & 0.05 \\
\hline ICS use & $18.9 \%$ & $9.5 \%$ & $<0.0001$ & $18.0 \%$ & $15.9 \%$ & 0.36 \\
\hline ICS-LABA combination use & $59.8 \%$ & $25.8 \%$ & $<0.0001$ & $58.9 \%$ & $49.3 \%$ & 0.12 \\
\hline Theophylline & $12.0 \%$ & $3.5 \%$ & $<0.0001$ & $12.0 \%$ & $8.6 \%$ & 0.06 \\
\hline \multicolumn{7}{|c|}{ COPD medications within 14 days after the index date } \\
\hline LABAs & $5.5 \%$ & $0.7 \%$ & $<0.0001$ & $4.6 \%$ & $3.3 \%$ & 0.28 \\
\hline LAMAs & $25.4 \%$ & $6.8 \%$ & $<0.0001$ & $23.1 \%$ & $22.0 \%$ & 0.66 \\
\hline ICS & $8.5 \%$ & $2.7 \%$ & $<0.0001$ & $8.3 \%$ & $6.8 \%$ & 0.34 \\
\hline ICS-LABA combination & $23.2 \%$ & $8.3 \%$ & $<0.0001$ & $22.3 \%$ & $23.1 \%$ & 0.74 \\
\hline Theophylline & $6.6 \%$ & $1.2 \%$ & $<0.0001$ & $6.6 \%$ & $5.2 \%$ & 0.35 \\
\hline
\end{tabular}

Abbreviations: COPD, chronic obstructive pulmonary disease; LABAs, long-acting $\beta_{2}$-agonists; LAMAs, long-acting muscarinic antagonists; ICS, inhaled corticosteroids.

\section{Publish your work in this journal}

The International Journal of COPD is an international, peer-reviewed journal of therapeutics and pharmacology focusing on concise rapid reporting of clinical studies and reviews in COPD. Special focus is given to the pathophysiological processes underlying the disease, intervention programs, patient focused education, and self management protocols.

\section{Dovepress}

This journal is indexed on PubMed Central, MedLine and CAS. The manuscript management system is completely online and includes a very quick and fair peer-review system, which is all easy to use. Visit $\mathrm{http}: / / \mathrm{www}$.dovepress.com/testimonials.php to read real quotes from published authors. 